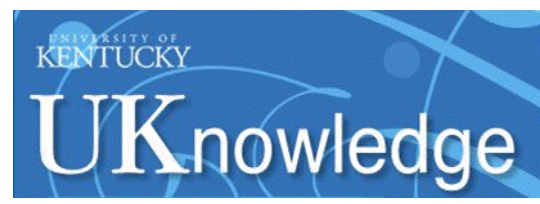

University of Kentucky

UKnowledge

\title{
Factors Influencing Final Outcomes in Patients with Shoulder Pain: A Retrospective Review
}

Timothy L. Uhl

University of Kentucky, tluhl2@uky.edu

Enrique V. Smith-Forbes

Fort Sam Houston Clinic, e_vsf12@hotmail.com

Arthur J. Nitz

University of Kentucky, arthur.nitz@uky.edu

Follow this and additional works at: https://uknowledge.uky.edu/rehabsci_facpub

Part of the Rehabilitation and Therapy Commons

Right click to open a feedback form in a new tab to let us know how this document benefits you.

\section{Repository Citation}

Uhl, Timothy L.; Smith-Forbes, Enrique V.; and Nitz, Arthur J., "Factors Influencing Final Outcomes in Patients with Shoulder Pain: A Retrospective Review" (2017). Physical Therapy Faculty Publications. 68. https://uknowledge.uky.edu/rehabsci_facpub/68

This Article is brought to you for free and open access by the Physical Therapy at UKnowledge. It has been accepted for inclusion in Physical Therapy Faculty Publications by an authorized administrator of UKnowledge. For more information, please contact UKnowledge@lsv.uky.edu. 


\section{Factors Influencing Final Outcomes in Patients with Shoulder Pain: A Retrospective Review}

Digital Object Identifier (DOI)

https://doi.org/10.1016/j.jht.2017.04.004

Notes/Citation Information

Published in Journal of Hand Therapy, v. 30, issue 2, p. 200-207.

(c) 2017 Hanley \& Belfus, an imprint of Elsevier Inc.

This manuscript version is made available under the CC-BY-NC-ND 4.0 license

http://creativecommons.org/licenses/by-nc-nd/4.0/. 
1 Factors Influencing Final Outcomes in Patients with Shoulder Pain: A Retrospective

2 Review 


\section{ABSTRACT:}

\section{Study Design:}

6 Retrospective cohort.

7 Introduction:

8 Rehabilitation interventions are commonly prescribed for patients with shoulder pain,

9 but it is unclear what factors may help clinicians' prognosis for final outcomes.

10 Purpose of the Study:

11 To determine what factors are the best predictors of improved patient-reported

12 outcomes at discharge in patients with shoulder pain.

13 Methods:

14 Retrospective chart review of 128 patients presenting with shoulder pain to an

15 outpatient physical therapy clinic. Chart review captured data regarding patient

16 demographics, treatment interventions, patient history, and patient-reported outcome

17 scores. The primary dependent variable was the overall change score of the

18 QuickDASH (initial - discharge). Thirty-eight predictor variables were entered into a

19 forward stepwise multivariate linear regression model to determine which variables and

20 to what degree contributed to the dependent variable.

\section{Results:}

22 The linear regression model identified five predictor variables that yielded an $\mathrm{R}=.74$

23 and adjusted $\mathrm{R}^{2}=.538(\mathrm{P}<.001)$. The five predictor variables identified in order of 
24 explained variance are QuickDASH change at the $5^{\text {th }}$ visit, a total number of visits, initial 25 QuickDASH score, scapular retraction exercise, and age.

26 Discussion: Early change scores, equal to minimal detectable change scores on

27 patient-reported outcomes appear to be strong indicators that patients with shoulder

28 pain are on a positive trajectory to benefit from rehabilitation.

29 Conclusion:

30 Using patient-reported outcomes throughout care, not just at the start and end of care,

31 will provide therapist feedback regarding patient's progress and indicate treatment

32 effectiveness.

33 Keywords: Patient-reported outcome, QuickDASH, physical therapy, prognosis

34 Levels of Evidence: Level: 4

35

36

37 


\subsection{Introduction:}

Shoulder pain is a common and debilitating diagnosis in health care with a 1-year prevalence reaching up to $47 \%$ in the adult population. ${ }^{1}$ It is second, only to low back

42 pain in prevalence of musculoskeletal conditions for those seeking care. ${ }^{2}$ Shoulder pain is often associated with unfavorable outcomes with roughly $40-50 \%$ of all new episodes of shoulder pain patients presenting to a primary care setting, who still report symptoms 6 to 12 months later. ${ }^{3-5}$ outcomes with rehabilitation intervention is growing but is inconsistent. A systematic education decreases pain and improves function at short-term and long-term follow-up search of the literature identified 16 studies that focused on the prognosis of shoulder disorders; only six, were designated as "high quality". ${ }^{6}$ Strong evidence exists that high pain intensity and middle age (45-54) are associated with poor outcomes. There is moderate evidence that prognostic factors for shoulder pain such as long duration of complaints, and high disability score at baseline predict a poor outcome in primary care. ${ }^{6-8}$ Many prognostic factors were considered in these 16 studies such as sex, mechanism of injury, psychological factors, work demands, anatomical factors and impairments of motion and strength. ${ }^{6}$ However the vast majority of the prognostic factors were identified only at baseline.

(1)

There is compelling evidence in the literature indicating exercise and patient for patients with impingement syndrome. ${ }^{9,10}$ Additional interventions such as 
61 mobilization, modalities, and stretching interventions are commonly used to treat

62 shoulder impingement syndrome with limited evidence to support their use. ${ }^{11}$

63 Clinicians are often faced with questions relating to identifying prognostic factors that

64 will determine a patient's positive outcome. Treatment approaches may vary based on

65 the presence or absence of a number of prognostic factors. There are a many factors

66 related to history, co-morbidities, psychological state, physical impairments, work

67 demands, physical examination findings and patient self-report perceptions of function

68

69

70

71

72 81 baseline. ${ }^{13}$ that can contribute to a patient's outcome. It is not known how much each of these items is weighted in contributing to a patient's prognosis.

The role of early response to rehabilitation has demonstrated limited evidence in predicting long-term outcomes. Researchers investigated the effects of early selfreported changes in levels of disability and pain levels in patients with acute back pain seeking chiropractic care. ${ }^{12}$ Axen et al. ${ }^{12}$ found that improvement in pain and disability after visit 2 increased the odds of a positive treatment outcome by an 2.9 odds ratio compared to patients with no improvement. This response was further investigated in 2422 patients presenting with multiple musculoskeletal conditions to chiropractors in the United Kingdom over an 8 year period..$^{13}$ The best predictor of a positive outcome at the tenth visit in those patients with persistent musculoskeletal pain was self-reported improvement by the fifth visit. These authors suggested that early changes may be more important as predictors in musculoskeletal conditions than variables measured at

It is hoped that the acquired knowledge of the prognostic factors that contribute to the successful outcomes of patients with shoulder pain will help provide more 
84 informed clinical decision-making among health care practitioners. The gained

85 information can be provided to patients regarding their plan of care and opportunity for a successful outcome. Based on the previous research we sought to be as inclusive as possible in retrospectively examining the charts plus our own clinical observations led us to incorporate the early treatment interventions and change scores of patient-self reports as prognostic factors accounting for the large number of factors used in this study. The objective of this study was to determine what factors are associated with a positive outcome in patients with shoulder pain presenting to a physical therapy clinic in a general orthopedic practice population. Specifically, we sought to identify which early interventions, historical presentation, and demographic variables are most associated with contributing to a positive outcome in patients presenting with shoulder pain.

\section{$\underline{2.0 \text { Methods }}$}

(1)

We conducted a retrospective study involving patients presenting to a single outpatient physical therapy clinic with shoulder pain between the years 2008-2010. The outpatient clinic was located in the southern region of the United States representing a typical general outpatient orthopedic clinic seeking care for shoulder pain. This clinic had instituted patient-reported outcome data collection as a standard operating procedure in the fall of 2006. This clinic used the QuickDASH to track disability scores for upper extremity injuries. Subject data was obtained by performing systematic chart reviews of patients with shoulder pain. Data was subsequently entered into an SPSS version 22 (IBM, Armonk, NY) for statistical analysis. The retrospective chart review was approved by the university's institution review board, IRB\# 10-0765-X1B before initiation of this study. 
The QuickDASH is a shortened version of the original disability of the arm, shoulder and hand (DASH) questionnaire. ${ }^{14}$ The QuickDASH is an 11 item disability scale ranging from 0 reflecting no disability to 100 indicating severe disability and has been found to be highly correlated with the full DASH. ${ }^{14}$ The QuickDASH has demonstrated excellent reliability in patients with shoulder pain $(I C C=0.90)$ with minimal detectable change $(\mathrm{MDC})=11.2$ and minimally clinically important differences $(\mathrm{MCID})=8$ in patients with shoulder pain. ${ }^{15}$

\section{$\underline{2.1 \text { Subjects }}$}

Two hundred eighty-nine patient charts were reviewed. Potential subjects were excluded from analysis if any of the following were present: a history of shoulder surgery $(n=12)$, neurological involvement $(n=26)$, shoulder instability $(n=6)$, severe loss of motion, suggesting adhesive capsulitis $(n=57)$ or a positive lag sign, suggesting rotator cuff tear $(n=1)$ and if the QuickDASH score at visit 5 was not recorded $(n=8)$. One hundred seventy-nine patient charts were available to extract data. Neurological involvement was identified by an abnormal dermatome, myotome or deep tendon reflex test. ${ }^{16}$ A positive apprehension sign, anterior or posterior drawer test was considered indicative of shoulder instability. ${ }^{17}$ Severe loss of motion was found to be present if the patient lacked greater than $50 \%$ of the normal physiologic range of motion. ${ }^{18}$ Finally, lag signs included a positive drop arm test, lift off test or external rotation lag sign. ${ }^{17}$

The terminology of shoulder pain was used in this study as this was a retrospective review and all patients were not screened with a standard evaluation for a particular pathology. We made an effort to exclude patients reporting history or physical 
examination findings consistent with instability, adhesive capsulitis, neurological involvement, and rotator cuff tears, presuming the remaining patients are likely to have rotator cuff impingement, the most common diagnosis in patients with shoulder pain. ${ }^{19,}$ 20 There is a strong bias that most of these patients had some level of rotator cuff inflammation or impingement which is challenging to classify. ${ }^{21}$ We were unable to confirm this diagnosis, therefore the terminology of shoulder pain was retained for this study.

\subsection{Chart Review Procedures}

Patient charts were systematically reviewed extracting information regarding exclusion criterion (5 variables), treatment received in first 5 visits (20 variables), treatment frequency (2 variables) demographical information (4 variables), comorbidities (4 variables), historical data (4 variables), physical examination (2 variables) and QuickDASH scores at three time points (initial, visit 5, and discharge). The dependent variable is the Overall QuickDASH change score which was calculated from the change between the initial and discharge QuickDASH scores. The QuickDASH change score at the $5^{\text {th }}$ visit was calculated as the difference between the initial and visit 5 QuickDASH scores. Nine continuous predictor variable were extracted: age, height, weight, duration of symptoms, pain levels, treatment frequency, the total number of treatments, initial QuickDASH score, change at $5^{\text {th }}$ visit QuickDASH score. Descriptive analysis of continuous data is presented with mean and 95\% confidence intervals in Table 1.

Nine binomial variables were extracted as present or absent and coded as 1 or 0 , respectively. The chart was reviewed for the presence of four comorbidities; history of 
151 cancer, use of tobacco, use of alcohol, history of diabetes. These were the primary

152 comorbidities filled out on the standard medical history form completed by patients at

153 intake. Additionally, four historical variables, previous injury in the shoulder and work-

154 related injury were coded on the excel spreadsheet. The last two binomial variables

155 coded were mechanism of injury atraumatic $=1$, and traumatic $=0$ and sex was coded

156 as 1 for males and 0 for females. The final variable from physical examination was the

157 presence of limited elevation which was coded as present if the chart record indicated

158 shoulder elevation less than $140^{\circ}$ on the involved shoulder. This value was used based

159 on previous research identifying $156 \pm 12^{\circ}$ as typical flexion available in patients with

160 shoulder pain. ${ }^{22}$ The value of 140 was decided upon based on measurement error

161 ranging from $3-7^{\circ}$ around $144^{\circ} .{ }^{22}$ The frequency count of all these potential predictor

162 variables are presented in Table 1.

\subsection{Treatment Variable Descriptions}

The therapist providing treatment recorded the specific modality, if applied, for each treatment and pertinent details of that application including number of minutes applied, specific anatomical site of application and numerical settings on the machine

167 where applicable which is the standard practice for this clinic and is useful for

168 subsequent treatments. The twenty treatment predictor variables were treated as continuous data ranging from 0 to 5 depending on the number of treatments received

170 during the first five visits for the purposes of this project. For example, if a patient

171 received an ultrasound three times during first five treatments they would be coded as

172 three in the ultrasound variable. A zero would be coded in a treatment variable if

173 nowhere in the notes was there an indication that a patient received a particular 
174 intervention. The mean, 95\% confidence interval, and frequency counts for each 175 treatment category are presented in Table 1. This study only recorded data from the

176 first five visits as our primary interest was how early interventions and early QuickDASH

177 change scores would affect final outcomes. Previous research had demonstrated

178 dramatic changes early during intervention, which was consistent with our clinical

179 observations. ${ }^{23}$

Treatment counts were used in this study as this was a retrospective study. The specific parameters of each treatment were not documented and varied between

182 therapists based on individual therapist clinical decisions for their patient. We chose to use treatment counts to document what occurs in a typical rehabilitation intervention for shoulder pain. Ideally, having a standard treatment protocol for all patients would have 185 strengthened the study but this would not represent what is typically occurring in outpatient physical therapy clinic with multiple therapists treating patients. Five

187 treatment modalities were recorded to indicate if a patient either did or did not receive 188 the intervention as part of their treatment for each of the five visits. The modality had to 189 be directed to the shoulder to be counted. Manual therapy interventions were 190 categorized into three variables. Spine mobilizations were treatment interventions 191 directed at cervical and thoracic joints regardless of the intensity of the mobilization.

192 Glenohumeral mobilization was manual therapy treatment to gain range of motion in 193 any direction of the shoulder regardless of the intensity. Soft-tissue mobilization was a 194 massage or myofascial release techniques directed at surrounding shoulder and 195 scapular musculature. Various exercises were prescribed so instead of specifying each 196 exercise we categorized exercises into eleven components based on mode and 
197 direction. The mode was either passive range of motion (PROM), active assistive range 198 of motion (AAROM), or resistive range of motion (RROM). The three primary directions 199 identified were flexion regardless of the plane of elevation, internal and external rotation 200 irrespective of the amount of arm abduction. Strengthening exercises at this clinic 201 focused on scapular exercises, so two additional categories of RROM were added for 202 scapular protraction and retraction: exercises representing scapular punches and 203 pinches using various resistive loads. Education regarding postural correction for 204 forward head or shoulders was counted as a postural exercise intervention.

When examining the data entered into the excel spreadsheet 51 subjects had at 206 least one missing predictor variable, therefore, these subject were removed, leaving 128 207 subjects available for the forward stepwise regression model. $<<$ Insert Table 1 $>>$

\subsection{Data Analysis}

A multivariate linear regression analysis was conducted to determine which of

211 the 38 predictor variables were most prognostic in estimating final outcome on the

212 QuickDASH score. The outcome or dependent variable (Y) was the overall QuickDASH

213 change score. The predictor variables were entered into a forward stepwise linear 214 regression using SPSS version 22 (IBM, Corporation, Armonk NY). A forward stepwise

215 method places the predictor variable with the highest correlated to the outcome variable 216 into the equation first. A p-value of $\leq .05$ was required for a predictor variable to enter 217 the equation at each step requiring that the addition of other predictor variables had to 218 contribute to estimating the outcome variable significantly. Additionally, at each step a 
$219 p$-value $\geq 0.1$ was used to remove a predictor variable that exceeded this value at each

220 step in developing the regression model. The adjusted $\mathrm{R}^{2}$ value was determined at

221 each step to evaluate the explained variance by the predictor variables. The variable

222 inflation factor was monitored at each step to assure predictor variables entered were

223 not highly correlated and identify potential multicollinearity between predictor variables

224 added to the equation.

\section{$225 \quad 3.0$ Results}

The multivariate linear regression analysis revealed a model with an adjusted $\mathrm{R}^{2}$

$227=.538(\mathrm{P}<.001)$ accounting for 5 variables entered into the equation. (Table 2$)$ The five

228 variables in order were QuickDASH change score at 5 visits, Total visits, Initial

229 QuickDASH score, RROM Scapular retraction, and Age. The resulting regression

230 equation was able to account for approximately half of the variance of the change in

231 perceived level of disability measured by the QuickDASH in patients with shoulder pain

232 with a standard error of the estimate equal to 10.5 points. The resulting equation:

$233 \mathrm{Y}($ Overall QuickDASH change score $)=.62\left(\right.$ QuickDASH change score at $5^{\text {th }}$ visit $)+$ 234.73 (Total visits) +.238 (Initial QuickDASH score) +1.22 (RROM scapular retraction) 235.165 (Age) -4.69.

$236<<$ Insert Table 2>>

\section{$237 \quad$ 4.0 Discussion}

The objective of this study was to determine what factors are associated with a 239 positive outcome in patients with shoulder pain presenting to a physical therapy clinic in 
240 a general orthopedic practice population. With the recent emphasis on value-based

241 health care ${ }^{24,25}$ and estimating final outcomes through the use of G-codes ${ }^{26}$ clinicians $^{2}$

242 need to establish and refine reasonable predictors for patient outcomes. The current

243 study predicts short-term outcomes, as indicated by patient self-reported outcomes

244 using the QuickDASH change scores and provides clinicians a potential tool to estimate

245 outcome change scores in patients with shoulder pain.

\subsection{Comparison to Previous Studies}

Previous studies have investigated short-term outcome predictors for patients with shoulder pain. A systematic review of shoulder disorders found strong evidence

249 that high pain intensity predicts poor outcomes in primary care populations. It also

250

251

252

253

254

255

256

257

258

259

260

261

262 revealed moderate evidence for long duration of complaints and high disability score at baseline. ${ }^{6}$ Similar findings were obtained in another systematic review, which found only two prognostic factors associated with outcome in two or more studies, duration of pain and baseline function. ${ }^{27}$ All of these measures were included in this study however our results differed. Baseline pain level was not predictive of an overall change of the QuickDASH score, and higher initial QuickDASH scores were associated with greater change scores. It is logical and commonly reported that higher scores are predictive of greater change as there is more opportunity for change. ${ }^{28}$ Based on previous studies we expected the duration of symptoms and pain levels to be predictive of change scores. ${ }^{6}$

27 These results were not observed in this study. The previous systematic reviews were primarily in prospective cohort studies and the current study is a retrospective cohort limiting the impact of this study's findings related to these two variables. In reviewing our data duration of symptoms ranged from very acute at .2 months to very chronic - 161 
263 months. However, duration of symptoms was skewed toward the acute end of the 264 continuum as $61 \%$ of all 128 subjects identified their duration of symptoms as less than 265 or equal to 4 months. This relatively tight distribution was observed for baseline pain 266 measures as $64 \%$ of all subjects rated their pain between 4 and 7 on a numeric pain 267 rating scale. The lack of variability for each of these predictor variables likely contributes 268 to their absence in the final regression model.

Previous studies investigating short-term outcomes of physical therapy lasting 3 270 months in duration found older age to predict greater disability at discharge. ${ }^{29,} 30$ The 271 use of age as a predictor is consistent with the results from the current study. We 272 identified age as a negative predictor, indicating the greater the age, the poorer our 273 outcome. However, this is a non-modifiable predictor variable and was a significant but 274 weak predictor of final outcome as it was the last variable included in the regression 275 model. Clinicians need to remember that in a regression model, it is the combination of 276 variables that create the model, rather than a single variable and the relative importance 277 is indicated by explained variance which in this case only contributed $1.5 \%$ of the 278 explained variance.

The current study supports previous research that identified incorporating active 280 interventions as prognostic of positive outcomes in patients with shoulder pain..$^{9,10}$ It is 281 interesting to note that no other intervention was strongly correlated with a positive 282 outcome other than resistive scapular retraction exercises. ${ }^{29} \mathrm{~A}$ substantial number of 283 the patients included in this study exhibited signs and symptoms of impingement 284 syndrome, though we did not specifically limit this study to patients with symptoms of 285 impingement alone. Because we excluded patients with significant motion restrictions 
and clinical findings consistent with shoulder instability, the majority of the remaining patients demonstrated rotator cuff involvement. Exercises focusing on scapular retraction provide benefit by increasing subacromial space ${ }^{31,32}$ and thereby reducing shoulder pain and dysfunction. ${ }^{23,33,34}$ Experienced therapists note that scapular exercise is a fundamental element in their treatment of patients with shoulder pain likely to have sub-acromial impingement. ${ }^{35}$

Another factor that was a positive predictor of overall change in QuickDASH score in the current study was the total number of therapy visits. Though the aim of any treatment protocol is to achieve established goals in as timely a manner as possible, this information suggests there is important value in ultimate patient outcome by persisting with a treatment regimen, even if progress occurs at a slow pace. Given this evidence, therapists should be encouraged to persist in pursuing treatment goals and continue to evaluate specific features of treatment interventions, especially working to see that patient's "buy in" to the value of disciplined compliance with the prescribed exercise regimen. ${ }^{35}$ In making this recommendation, it is recognized that beyond some, as yet, unidentified upper threshold of visit number, the positive predictive characteristic indicated by our research probably fades in value. We did not seek to establish that upper limit of visits as that was not the focus of the current study.

The results of the current study provide treating clinicians with useful information to track patient progress and modify treatment interventions based on that progress. The variable in our study most closely associated with predicting a successful patient response was the QuickDASH change score at the $5^{\text {th }}$ therapy visit. This variable by itself was capable of explaining $40 \%$ of the variance of the overall change in the 
QuickDash score at discharge. Tate et al., ${ }^{23}$ demonstrated graphically in their report that patients with shoulder impingement who exhibited significant improvement had at 2 weeks improved by approximately 15 points. Their sample was only 10 patients, but our findings agree and support their conclusions and a similar trend of early improvement in patient-reported outcomes. This is consistent with the current study as the average improvement of all patients in our study was 11 points improvement with a $95 \%$ confidence interval ranging from 9-13 points. This meets and exceeds the minimally clinically important difference of 8 points identified as meaningful improvement in patients with shoulder pain. ${ }^{15}$ The use of patient-reported outcomes throughout the course of care, and not just at the beginning and end of treatment, is valuable as indicated by the results of the current study. This is important as it allows therapists the opportunity to adjust therapeutic intervention in an effort to prevent protracted pain and disability when patients do not self-report clinically meaningful functional progress. Failure to note substantial progress during the first five therapy visits should alert clinicians to examine the range of interventions for any given patient. ${ }^{13}$

\subsection{Clinical Implications}

The linear regression equation created from this dataset provides clinicians with a tool to estimate QuickDASH change score at discharge. This equation indicates that all variables are positive predictors of the overall QuickDASH change score except for age. The age variable has a negative sign indicating that for every year older the overall QuickDASH change score is decreased by .16 units. Any value can be applied to estimate the overall change in QuickDASH score. For the following example, the mean values for each predictive variable from Table 1 were used. 
QuickDASH change score at $5^{\text {th }}$ visit $=11$

Total visits $=12$

Initial QuickDASH score $=40$

RROM scapular retraction $=4$

Age $=50$

Equation $(.62(11)+.73(12)+.238(40)+1.22(4)-.165(50)-4.69)=17$

Applying the regression equation presented in the results section with mean values yields an estimated change score equal to 17 points. In applying this equation,

341 the standard error of estimate, which equals 10.5 also has to be taken into

342 consideration. The standard error of estimate for a regression equation functions similar to a standard deviation of the mean, which would indicate that change score could vary

344 from 6.5 to 27.5 points. The wide range of scores is explained by the fact that the 345 equation only explains approximately $50 \%$ of the variance of the overall QuickDASH 346 change score and indicates that other factors not considered may contribute to the final 347 outcome. This equation is not a perfect estimator of final change score but provides 348 clinicians with the major predictor variables to consider and a useful tool to estimate 349 prognosis.

Through examining the data from a different perspective, clinicians should focus

351 on achieving an eleven point change or greater in the first five visits. The data was

352 further evaluated to determine how likely a change of 11 points at visit 5 predicts a 
353 QuickDASH change score of 17 or greater at discharge. Fifty-five out of the 128 patients

354 had an overall QuickDASH change score greater than 17. Forty-one out of 55 or $74.5 \%$

355 of those patients had a change of 11 or more points on the QuickDASH at visit 5 . This

356 left 73 patients with an overall QuickDASH change score under 17 points. Fifty-three out

357 of 73 or $72.6 \%$ of those patients had a QuickDASH change score under 11 points at

358 visit 5 . This change value has been found to be the minimal detectable change score

359 with the QuickDASH in patients with shoulder pain. ${ }^{15}$ This demonstrates that early use

360 of patient-reported outcomes provide clinicians with the patient's perspective of how

361 they are recovering from their shoulder injury and is essential in patient-centered

362 evidence-based rehabilitation. ${ }^{36-39}$

\subsection{Limitations}

A number of limitations exist in the present study. This is a retrospective study which has a common limitation of missing data. This study is no different. A lack of objective data in the chart reviews relating to inclusion criteria limited our ability to

367 diagnose patients with a specific condition such as subacromial impingement syndrome.

368 It is very challenging, and much debate is ongoing in the literature regarding how to categorize these patients. ${ }^{21,40} \mathrm{We}$ attempted to include potential patients by using reasonably strict exclusion criterion to limit our patient population, since advanced

371 imaging was not available for all patients during this retrospective review of physical

372 therapy notes. As a retrospective study all patients information is derived from either 373 self-report or from therapist reported findings. There are details that could have

374 contributed to the explaining final outcomes that were not able to be obtained or coded 375 in a manner to allow adequate investigation such as continuous measures of motion 
and strength measures in a standard manner. Future studies may be designed to create a more stringent inclusion criteria related to diagnosing sub-acromial impingement and using a prospective, rather than a retrospective design.

Another significant limitation refers to the generalizability of the results. The study was conducted in a single outpatient facility in the south region of the United States with only four treating therapists. It is unknown whether the results of our study will apply to other patient populations in different clinical settings. Future research should seek to replicate the present study in various regions of the country with a different cohort of patients to improve the generalizability of these results. Additionally, the specific intervention parameters were not well defined in this study. For example, it is not clear what aspects of the joint capsule were targeted with glenohumeral mobilization. More accurate treatment descriptions in a prospective, more controlled study may assist future therapists in determining specific treatment plans for their patients. Finally, the number of patients included in this study $(n=128)$ was relatively low considering the number of variables considered in the regression model and may have led to less reliable results. Future research would benefit from including a larger number of patients in a similar study.

\section{$\underline{4.4 \text { Conclusion }}$}

The goal of this study was to identify the best predictors of patient-reported outcome change scores at discharge in patients with shoulder pain. We identified four positive predictive factors with the greatest predictor being QuichDASH change score at visit 5 using a retrospective design. The other three positive predictive factors were the 
398 number of patient visits, initial QuickDASH score, and incorporation of scapular

399 retraction resistive exercises. The only negative predictor was the age of the patient,

400 which is not modifiable. A change score of 11 points, equivalent to the minimal

401 detectable change score for the QuickDASH, early in rehabilitation is a positive

402 indication that patients with shoulder pain are on a positive trajectory to achieving a

403 good outcome with rehabilitation. 


\subsection{References}

1. Luime JJ, Koes BW, Hendriksen IJM, et al. Prevalence and incidence of shoulder pain in the general population; a systematic review. Scandinavian Journal Of Rheumatology. 2004;33:73-81.

2. Pope DP, Croft PR, Pritchard CM, Silman AJ. Prevalence of shoulder pain in the community: the influence of case definition. Ann Rheum Dis. 1997;56:308-312.

3. Croft P, Pope D, Silman A. The clinical course of shoulder pain: prospective cohort study in primary care. Primary Care Rheumatology Society Shoulder Study Group. Bmj. 1996;313:601-602.

4. van der Windt DA, Koes BW, de Jong BA, Bouter LM. Shoulder disorders in general practice: incidence, patient characteristics, and management. Annals Of The Rheumatic Diseases. 1995;54:959-964.

5. Winters JC, Sobel JS, Groenier KH, Arendzen JH, Meyboom-de Jong B. The long-term course of shoulder complaints: a prospective study in general practice. Rheumatology (Oxford). 1999;38:160-163.

6. Kuijpers T, van der Windt DIAWM, van der Heijden GJMG, Bouter LM. Systematic review of prognostic cohort studies on shoulder disorders. Pain. 2004;109:420-431.

7. Mallen CD, Peat G, Thomas E, Dunn KM, Croft PR. Prognostic factors for musculoskeletal pain in primary care: a systematic review. Br J Gen Pract. 2007;57:655-661. 
427 8. Feleus A, Bierma-Zeinstra SM, Miedema HS, et al. Prognostic indicators for nonrecovery of non-traumatic complaints at arm, neck and shoulder in general practice--6 months follow-up. Rheumatology (Oxford). 2007;46:169-176.

9. Hanratty CE, McVeigh JG, Kerr DP, et al. The effectiveness of physiotherapy exercises in subacromial impingement syndrome: a systematic review and metaanalysis. Semin Arthritis Rheum. 2012;42:297-316.

10. Brox Jl, Brevik Jl. Prognostic factors in patients with rotator tendinosis (stage II impingement syndrome) of the shoulder. Scandinavian Journal Of Primary Health Care. 1996;14:100-105.

11. Struyf F, De Hertogh W, Gulinck J, Nijs J. Evidence-based treatment methods for the management of shoulder impingement syndrome among Dutch-speaking physiotherapists: an online, web-based survey. J Manipulative Physiol Ther. 2012;35:720-726.

12. Axen I, Rosenbaum A, Robech R, Larsen K, Leboeuf-Yde C. The Nordic back pain subpopulation program: can patient reactions to the first chiropractic treatment predict early favorable treatment outcome in nonpersistent low back pain? Journal Of Manipulative And Physiological Therapeutics. 2005;28:153-158.

13. Bolton JE, Hurst HC. Prognostic factors for short-term improvement in acute and persistent musculoskeletal pain consulters in primary care. Chiropractic \& Manual Therapies. 2011;19:27-27.

14. Gummesson C, Ward MM, Atroshi I. The shortened disabilities of the arm, shoulder and hand questionnaire (QuickDASH): validity and reliability based on 
responses within the full-length DASH. BMC musculoskeletal disorders.

450

451

452

453

454

455

456

457

458

459

460

461

462

463

464

465

466

467

468

469 2006;7:44.

15. Mintken PE, Glynn P, Cleland JA. Psychometric properties of the shortened disabilities of the Arm, Shoulder, and Hand Questionnaire (QuickDASH) and Numeric Pain Rating Scale in patients with shoulder pain. J Shoulder Elbow Surg. 2009;18:920-926.

16. Wainner RS, Fritz JM, Irrgang JJ, Boninger ML, Delitto A, Allison S. Reliability and diagnostic accuracy of the clinical examination and patient self-report measures for cervical radiculopathy. Spine. 2003;28:52-62.

17. Hegedus EJ, Goode A, Campbell S, et al. Physical examination tests of the shoulder: a systematic review with meta-analysis of individual tests. Br J Sports Med. 2008;42:80-92; discussion 92.

18. Kelley MJ, Shaffer MA, Kuhn JE, et al. Shoulder pain and mobility deficits: adhesive capsulitis. J Orthop Sports Phys Ther. 2013;43:A1-31.

19. Bamji AN, Erhardt CC, Price TR, Williams PL. The painful shoulder: can consultants agree? Br J Rheumatol. 1996;35:1172-1174.

20. Bigliani LU, Levine WN. Subacromial impingement syndrome. J.Bone.Joint.Surg.Am. 1997;79:1854-1868.

21. de Witte PB, de Groot JH, van Zwet EW, et al. Communication breakdown: clinicians disagree on subacromial impingement. Med Biol Eng Comput. 2014;52:221-231. 
22. Mullaney MJ, McHugh MP, Johnson CP, Tyler TF. Reliability of shoulder range of motion comparing a goniometer to a digital level. Physiotherapy Theory And Practice. 2010;26:327-333.

23. Tate AR, McClure PW, Young IA, Salvatori R, Michener LA. Comprehensive impairment-based exercise and manual therapy intervention for patients with subacromial impingement syndrome: a case series. J Orthop Sports Phys Ther. 2010;40:474-493.

24. Fritz JM. Physical therapy in a value-based healthcare world. J Orthop Sports Phys Ther. 2012;42:1-2.

25. Porter ME. What Is Value in Health Care?. New Engl J Med. 2010;363:24772481.

26. Register F. Centers for Medicare and Medicaid Services: Department of Health and Human Services; 2012.

27. Chester R, Shepstone L, Daniell H, Sweeting D, Lewis J, Jerosch-Herold C. Predicting response to physiotherapy treatment for musculoskeletal shoulder pain: a systematic review. BMC Musculoskel. Disord. 2013;14:203-203.

28. Hays RD, Woolley JM. The concept of clinically meaningful difference in healthrelated quality-of-life research. How meaningful is it? Pharmacoeconomics. 2000;18:419-423.

29. van der Heijden GJ. Shoulder disorders: a state-of-the-art review. Bailliere's best practice \& research. Clinical rheumatology. 1999;13:287-309. 
30. Kennedy CA, Manno M, Hogg-Johnson S, et al. Prognosis in soft tissue disorders of the shoulder: predicting both change in disability and level of disability after treatment. Physical Therapy. 2006;86:1013-1032.

31. Solem-Bertoft E, Nordin M, Rahme H, Westerberg CE. The hand in neck manoeuvre as a toll to analyze pain-generating mechanisms in the subacromial impingement syndrome. Clinical Orthopaedics and Related Research. 1994;26:59-64.

32. Seitz AL, McClure PW, Lynch SS, Ketchum JM, Michener LA. Effects of scapular dyskinesis and scapular assistance test on subacromial space during static arm elevation. J Shoulder Elbow Surg. 2012;21:631-640.

33. De Mey K, Danneels L, Cagnie B, Cools AM. Scapular muscle rehabilitation exercises in overhead athletes with impingement symptoms: effect of a 6-week training program on muscle recruitment and functional outcome. The American journal of sports medicine. 2012;40:1906-1915.

34. McClure PW, Bialker J, Neff N, Williams G, Karduna A. Shoulder function and 3dimensional kinematics in people with shoulder impingement syndrome before and after a 6-week exercise program. Phys Ther. 2004;84:832-848.

35. Hanratty CE, Kerr DP, Wilson IM, et al. Physical Therapists' Perceptions and Use of Exercise in the Management of Subacromial Shoulder Impingement Syndrome: Focus Group Study. Phys Ther. 2016;96:1354-1363.

36. Jaeschke RZ, Guyatt GH, Sackett DL. Users' guides to the medical literature III how to use an article about a diagnostic test are the results of the study valid? Journal of the American Medical Association. 1994;271:389-391. 
514 37. Sackett DL, Rosenberg WMC, Gray JAM, Haynes RB, Richardson WS. Evidence 515

38. MacDermid J, Michlovitz S. Incorporating outcome measures into evidencebased practice. In: Law M, MacDermid J, eds. Evidence-Based Rehabilitation. 2nd ed. Thorofare: Slack; 2008:63-94.

519 39. MacDermid JC. An introduction to evidence-based practice for hand therapists. J 520 Hand Ther. 2004;17:105-117.

521 40. Braman JP, Zhao KD, Lawrence RL, Harrison AK, Ludewig PM. Shoulder impingement revisited: evolution of diagnostic understanding in orthopedic surgery and physical therapy. Med Biol Eng Comput. 2014;52:211-219. 

Table 1. Description of data extracted from 128 patients' charts. Continuous data presented with means, standard deviation and $95 \%$ confidence intervals of the means. Binomial data is presented as frequency counts.

\begin{tabular}{|c|c|c|c|c|c|c|c|}
\hline Data Category & Variable & Mean & $\begin{array}{l}\text { Standard } \\
\text { Deviation }\end{array}$ & $\begin{array}{l}95 \% \mathrm{Cl} \\
\text { Lower } \\
\text { Boundary }\end{array}$ & $\begin{array}{l}95 \% \mathrm{Cl} \\
\text { Upper } \\
\text { Boundary }\end{array}$ & Present & Absent \\
\hline $\begin{array}{l}\text { Dependent } \\
\text { Variable }\end{array}$ & $\begin{array}{l}\text { Overall Change } \\
\text { QuickDASH score }\end{array}$ & 16.72 & 15.54 & 14.03 & 19.42 & & \\
\hline Comorbidities & Cancer & & & & & 6 & 122 \\
\hline Comorbidities & Alcohol & & & & & 94 & 34 \\
\hline Comorbidities & Tobacco & & & & & 18 & 110 \\
\hline Comorbidities & Diabetes & & & & & 15 & 113 \\
\hline Dash Score & Initial QuickDash score & 39.81 & 16.55 & 36.95 & 42.68 & & \\
\hline Dash Score & $\begin{array}{l}\text { QuickDash change } \\
\text { score at } 5^{\text {th }} \text { visit }\end{array}$ & 11.10 & 12.91 & 8.87 & 13.34 & & \\
\hline Demographics & Age & 52.83 & 12.23 & 50.71 & 54.95 & & \\
\hline Demographics & Sex & & & & & 74 females & 54 males \\
\hline Demographics & Height (cm) & 170.51 & 10.59 & 168.68 & 172.34 & & \\
\hline Demographics & Weight (kg) & 83.79 & 21.68 & 80.04 & 87.55 & & \\
\hline History & Mechanism & & & & & 90 atraumatic & 38 traumatic \\
\hline History & $\begin{array}{l}\text { Duration of symptoms } \\
\text { (months) }\end{array}$ & 7.61 & 17.02 & 4.66 & 10.56 & & \\
\hline History & Work related & & & & & 18 & 110 \\
\hline History & Previous injury & & & & & 39 & 89 \\
\hline Physical Exam & $\begin{array}{l}\text { Limited shoulder } \\
\text { elevation }\end{array}$ & & & & & 79 & 49 \\
\hline Physical Exam & Pain (NPRS) & 5.60 & 2.09 & 5.24 & 5.96 & & \\
\hline Treatment & IFC & 0.59 & 1.61 & 0.31 & 0.87 & 16 & 112 \\
\hline Treatment & TENS & 1.97 & 2.40 & 1.55 & 2.39 & 55 & 73 \\
\hline Treatment & Muscle stimulation & 1.45 & 2.24 & 1.06 & 1.84 & 39 & 89 \\
\hline
\end{tabular}




\begin{tabular}{|c|c|c|c|c|c|c|c|}
\hline Treatment & Iontophoresis & 3.31 & 2.28 & 2.92 & 3.71 & 89 & 39 \\
\hline Treatment & Ultrasound & 3.76 & 2.12 & 3.39 & 4.12 & 100 & 28 \\
\hline Treatment & Spine Mobilization & 0.63 & 1.57 & 0.36 & 0.90 & 20 & 108 \\
\hline Treatment & $\begin{array}{l}\text { Glenohumeral } \\
\text { mobilization }\end{array}$ & 3.73 & 1.73 & 3.43 & 4.03 & 111 & 17 \\
\hline Treatment & Soft tissue mobilization & 0.72 & 1.66 & 0.43 & 1.01 & 22 & 106 \\
\hline Treatment & PROM flexion & 0.64 & 1.57 & 0.37 & 0.91 & 21 & 107 \\
\hline Treatment & PROM external rotation & 2.57 & 2.25 & 2.18 & 2.96 & 80 & 48 \\
\hline Treatment & PROM internal rotation & 1.25 & 2.00 & 0.90 & 1.60 & 40 & 88 \\
\hline Treatment & AAROM flexion & 2.45 & 2.29 & 2.05 & 2.84 & 73 & 55 \\
\hline Treatment & $\begin{array}{l}\text { AAROM external } \\
\text { rotation }\end{array}$ & 0.38 & 1.23 & 0.17 & 0.60 & 13 & 115 \\
\hline Treatment & AAROM internal rotation & 0.10 & 0.67 & -0.02 & 0.22 & 3 & 125 \\
\hline Treatment & RROM internal rotation & 2.41 & 2.26 & 2.02 & 2.81 & 75 & 53 \\
\hline Treatment & RROM external rotation & 2.73 & 2.18 & 2.35 & 3.10 & 87 & 41 \\
\hline Treatment & $\begin{array}{l}\text { RROM scapular } \\
\text { protraction }\end{array}$ & 1.00 & 1.76 & 0.69 & 1.31 & 38 & 90 \\
\hline Treatment & $\begin{array}{l}\text { RROM scapular } \\
\text { retraction }\end{array}$ & 4.13 & 1.70 & 3.83 & 4.42 & 112 & 16 \\
\hline Treatment & RROM flexion & 1.01 & 1.85 & 0.69 & 1.33 & 34 & 94 \\
\hline Treatment & Posture exercises & 1.50 & 2.18 & 1.12 & 1.88 & 43 & 85 \\
\hline $\begin{array}{l}\text { Treatment } \\
\text { frequency }\end{array}$ & Total visits & 12.18 & 5.83 & 11.17 & 13.19 & & \\
\hline $\begin{array}{l}\text { Treatment } \\
\text { frequency }\end{array}$ & Visits per week & 2.08 & 0.60 & 1.97 & 2.18 & & \\
\hline
\end{tabular}

$\mathrm{Cl}=$ Confidence Interval

NPRS = numerical pain rating scale

Present $=$ number of people in the sample that received the treatment or had the condition 
Absent $=$ number of people in the sample that did not receive the treatment or did not have the condition

$\mathrm{Rx}=$ Treatment category

$\mathrm{IFC}=$ Interferential current

TENS = Transcutaneous electrical nerve stimulation

$\mathrm{PROM}=$ Passive range of motion

AAROM = Active assistive range of motion

$\mathrm{RROM}=$ Resistive range of motion 
Table 2. Regression analysis results with predictor variables present in order of entry into the forward stepwise regression model

\begin{tabular}{|c|c|c|c|c|c|c|c|}
\hline Variable & $\begin{array}{l}R \\
\text { value }\end{array}$ & $\begin{array}{l}\text { Adjusted } \\
\mathrm{R}^{2}\end{array}$ & Beta & $\begin{array}{l}\text { Beta Cl95 } \\
\text { Lower } \\
\text { Boundary }\end{array}$ & $\begin{array}{l}\text { Beta Cl95 } \\
\text { Upper } \\
\text { Boundary }\end{array}$ & Significance & VIF \\
\hline \multicolumn{8}{|l|}{ QuickDash } \\
\hline $\begin{array}{l}\text { change } \\
\text { score at } \\
5^{\text {th }} \text { visit }\end{array}$ & .636 & .400 & .620 & .459 & .780 & .000 & 1.24 \\
\hline $\begin{array}{l}\text { Total visits } \\
\text { Initial }\end{array}$ & .690 & .468 & .730 & .402 & 1.058 & .000 & 1.062 \\
\hline $\begin{array}{l}\text { QuickDash } \\
\text { score } \\
\text { RROM }\end{array}$ & .723 & .510 & .238 & .108 & .361 & .000 & 1.266 \\
\hline $\begin{array}{l}\text { scapular } \\
\text { retraction }\end{array}$ & .735 & .525 & 1.22 & .100 & 2.345 & .033 & 1.061 \\
\hline Age & .746 & .538 & -.165 & -.318 & -.013 & .034 & 1.01 \\
\hline \multicolumn{8}{|c|}{$\mathrm{R}=$ regression correlation values } \\
\hline \multicolumn{8}{|c|}{ VIF $=$ Variable inflation factor } \\
\hline $\mathrm{Cl}=$ & e & & & & & & \\
\hline
\end{tabular}

